\title{
Safety and Hemostatic Effectiveness of SURGICEL® Powder (Oxidized Regenerated Cellulose) in Controlling Mild or Moderate Parenchymal or Soft- Tissue Intraoperative Bleeding
}

Nawwar Al-Attar ( $\nabla$ Nawwar.Al-Attar@gjnh.scot.nhs.uk)

Department of Cardiothoracic Surgery, Golden Jubilee National Hospital, Glasgow, UK

Eric de Jonge

Ziekenhuis Oost-Limburg Genk

Richard Kocharian

Ethicon, Inc., 1000 US Highway 202 South, Raritan, NJ 08869 USA

Bogdan Ilie

Ethicon, Inc., 1000 US Highway 202 South, Raritan, NJ 08869 USA

Ellie Barnett

Ethicon, Inc, LIVINGSTON

Frederik Berrevoet

University Hospital Ghent

\section{Research Article}

Keywords: Surgery, bleeding, hemostasis, topical hemostat, absorbable topical hemostat, oxidized regenerated cellulose, SURGICEL $®$, hemostatic powder, patient-blood management

Posted Date: January 20th, 2022

DOI: https://doi.org/10.21203/rs.3.rs-1185642/v1

License: (a) (i) This work is licensed under a Creative Commons Attribution 4.0 International License. Read Full License 


\section{Abstract}

Background. Topical hemostats reduce blood loss and optimize patient outcome and health care costs. The choice of agent depends on mode of action and surgical situation. This post-market clinical study evaluated the safety and effectiveness of Surgicel $₫-P$, a powdered form of oxidized regenerated cellulose, designed to help control mild-to-moderate bleeding on broad or raw tissue surfaces.

Methods. In a prospective, single-arm, multicenter trial, adult subjects with a target bleeding site (TBS) (mild-to-moderate bleeding for which conventional hemostatic methods were impractical or ineffective) were treated with Surgicel®-P. The primary endpoint was the proportion of subjects showing hemostasis at 5 minutes after application, without re-bleeding needing other hemostatic treatment. Secondary endpoints were these proportions at 3 and 10 minutes. The investigators' ease-of-use evaluation of Surgicel $\circledast$ - $P$ was recorded via questionnaire. Safety endpoints were the incidences of re-bleeding and thromboembolic events through day 30 , and serious adverse events requiring surgical intervention through month 6 with a causal (including unlikely or at least possible) relationship to Surgicel®-P.

Results. In 8 centers, 103 subjects were enrolled [median(range) age 64.0(33-88) years], of which 100 completed the study. Two deaths occurred, due to causes unrelated to Surgicel®-P. Surgeries were open (53.4\%) or laparoscopic/thoracoscopic (46.6\%), and mostly urological (37.9\%) and abdominal (32.0\%); TBSs included various tissue types, with mild $(69.9 \%)$ or moderate $(30.1 \%)$ bleeding over a median(range) surface area of $4(0.02-72.0) \mathrm{cm}^{2}$. The 5 -minute hemostatic success rate (primary endpoint) was $87.4 \%$; the 3- and 10-minute rates (secondary endpoints) were $77.7 \%$ and $92.2 \%$, respectively. Subgroup analyses showed 3-, 5- and 10-minute rates of $84.7 \%, 93.1 \%, 95.8 \%$ for mild, versus $61.3 \%, 74.2 \%, 83.9 \%$ for moderate bleeding, and $80.0 \%, 90.9 \%, 96.4 \%$ for open versus $75.0 \%, 83.3 \%, 87.5 \%$ for laparoscopic/thoracoscopic procedures. Within 10 minutes, initial manual compression (27.5\%) was progressively abandoned, and additional Surgicel ${ }^{\circledR}-\mathrm{P}$ was applied to $17.4 \%$ of TBSs. Investigators reverted to suture, cautery or other topical hemostats in $6.8 \%$ of procedures. No safety signals were identified. Investigators favorably evaluated the ease of use of Surgicel®-P device.

Conclusions. This post-market clinical study supports the safety and efficacy of Surgicel®-P in controlling mild-to-moderate surgical bleeding in a broad range of surgical procedures.

Clinical Trial Registration number. NCT03762200, 03/12/2018

\section{Background}

Topical hemostatic agents have acquired an important role in the modern surgical management of perioperative bleeding. $[1,2]$ Uncontrolled surgical bleeding is responsible for a substantial clinical and health care resource utilization burden, specifically given population ageing and the associated increased prevalence of comorbidities and use of anticoagulants.[3-7] By facilitating hemostasis in situations of mild-to-moderate bleeding where conventional hemostatic measures are insufficient or impractical, the adjunctive use of topical hemostatic agents reduces blood loss and the need for systemic hemostatic 
drugs and blood transfusions. By reducing surgical time and surgical complications, they ultimately optimize patient health and health care costs. $[2,8,9]$ A variety of absorbable topical hemostatic agents are available which differ in physicochemical properties and mode of action, as well as ease of use, safety, and cost. The optimal choice of agent depends on the surgical scenario and bleeding situation (visibility, anatomy and access) and on the patient's coagulation and comorbidity status.[1, 10] The current armamentarium distinguishes mechanical topical agents which provide a structural matrix for hemostasis and are considered the easiest, safest and most economical to use, active agents which rely on human, bovine or recombinant thrombin, gelatin-thrombin matrix generically termed flowables, and sealants which are based on human fibrinogen or (semi)synthetic compounds.[1]

Oxidized regenerated cellulose (ORC) is a plant-based polymer, consisting of continuous fibers with mechanical hemostatic properties, that was first introduced as a hemostat in 1943 but is now available in various topical hemostatic formulations.[11, 12] ORC provides a matrix for clot formation and enhances platelet activation and adhesion. In addition, because it creates a local acidic milieu, ORC produces coagulative necrosis with a tamponade effect, and exerts local antimicrobial action.[13] As absorbable mechanical hemostats, ORC-based agents are suitable for use in patients with an intact coagulation system. They have been shown effective in facilitating hemostasis in randomized controlled trials in various open, laparoscopic and endoscopic surgery settings, and are one of the most frequently used mechanical hemostats. $[1,10]$ SURGICEL $\circledast$ Original and its family of products were developed to aid in controlling continuous surgical oozing in distinct surgical bleeding situations, and their performance and safety profile are supported by clinical studies in a wide spectrum of surgical procedures.[14] The recently marketed powdered delivery form of these products, Surgicel® Powder (Surgicel®-P), was designed to aid in mild-to-moderate intensity bleeding in a range of tissue surfaces including broad or raw surfaces and in locations where access or placement of fabric ORC products may be challenging or impractical. [15] Composed of aggregates of ORC fine fibers, Surgicel $\circledast-P$ has superior hemostatic efficacy compared to the constituent ORC fine fibers because of more favorable surface energetics and surface area.

[16] Studies with liver punch biopsy and liver abrasion in swine, which are acute experimental models of mild-to-moderate bleeding and mild diffuse oozing, respectively, Surgicel $\circledast$-P has shown superior hemostatic efficacy and faster time-to-hemostasis compared to other commercially available polysaccharide absorbable hemostats.[17] In addition, in the porcine liver abrasion model, Surgicel ${ }^{\circledR}-\mathrm{P}$ provided more effective and sustained hemostasis and faster time-to-hemostasis than a combination powder hemostat consisting of thrombin, collagen and chondroitin sulphate.[14] Surgicel ${ }^{\circledR}-\mathrm{P}$ combines the ORC powder formulation with a delivery system suitable for either open or laparoscopic/thoracoscopic approaches, that is particularly useful for large surfaces and difficult-toaccess anatomical locations where application of other forms of topical hemostats may be impractical. [15]

Here we present a post-market clinical study designed to further evaluate the safety and hemostatic effectiveness of Surgicel $\circledast$-P in controlling mild or moderate parenchymal or soft-tissue intraoperative bleeding in a clinical practice setting of general, gynecological, urological, and cardiothoracic surgery. 


\section{Methods}

\section{Study Design}

This was a prospective, single arm, multicenter trial on the adjunctive use of Surgicel®-P to control surgical hemorrhage (capillary, venous or small-arterial) for which conventional hemostatic methods are impractical or ineffective. The trial was designed as a post-marketing study across multiple surgical subspecialties and across open, laparoscopic or thoracoscopic procedures in adult subjects with a mild to moderate 'target bleeding site' (TBS). The TBS was defined as the first active bleeding site that was identified during surgical dissection, that was related to the primary operative procedure, and that required an adjunctive hemostat. The study intended to include at least 100 evaluable subjects from approximately 8 investigational sites (up to 20 subjects per site) in the United Kingdom and Belgium. The study was performed in accordance with the ICH tripartite guideline for Good Clinical Practice (1996) and the Declaration of Helsinki (2013). Protocols and informed consent forms were approved by institutional review boards at participating sites (WoSRES (West of Scotland Research Ethics Service) (Paisley, United Kingdom), Commissie Voor Medische Ethiek (Gent, Belgium), Ziekenhuis Oost Limburg Ethisch comité (Genk, Belgium).

\section{Study Subjects and Procedure}

Patients aged 18 years or older who required a non-emergent, open or endoscopic, and general, gynecological, hepatopancreatobiliary, cardiothoracic, or urological surgical procedure were considered for enrollment if informed consent was obtained and an INR $<1.5$ was documented within 24 hours of starting surgery. Subjects were included in the study if an appropriate TBS was identified intra-operatively and if none of the exclusion criteria were met (Supplementary information 1). These included: residual anticoagulant medication (except aspirin) or antiplatelet/P2Y12 inhibitor medication beyond the appropriate pre-operative wash-out period; any pre- or intra-operative finding that precluded the use of the study product; major arterial or venous bleeding or defect and/or risk of introducing study product into an open blood vessel; a TBS where silver nitrate or any other escharotic chemicals had been applied; a TBS in, around, or in proximity to foramina in bone, or areas of bony confine, the spinal cord, or optic nerve and chiasm; a TBS in urological procedures where study product could risk blocking the urethra, ureter or a catheter.

For each subject, two Surgicel®-P kits were available in the operating room, made ready-to-use prior to TBS identification. The Surgicel $\circledast$-P single-use applicator and angling tip were used in open procedures, and the Surgicel $\circledast$-P Endoscopic Applicator in laparoscopic and thoracoscopic procedures. After Surgicel ${ }^{\circledR}$-P application, the TBS was assessed for hemostasis at 3, 5, and 10 minutes, and just prior to initiating fascial closure in open procedures or to port-site closure in laparoscopic and thoracoscopic procedures. If hemostasis could not be achieved, re-application of Surgicel $®-P$ was allowed during the 10-minute assessment, and, if necessary, also other hemostatic measures. Post-operatively, subjects were 
followed until hospital discharge, and evaluated for adverse events (AEs) at $30(+14)$ days clinically, and for serious adverse events (SAE) related to SURGICEL $®-P$ and requiring surgical intervention at 6 months $( \pm 30$ days) clinically or by voice call.

Each investigator was asked to complete an Ease-of-Use survey for their first 2 open and first 2 endoscopic procedures performed within the study. Questionnaires were to be completed as soon as possible, but within approximately 72 hours.

\section{Study Endpoints}

The primary endpoint was the proportion of subjects achieving hemostatic success at 5 minutes, which was defined as hemostatic control at the TBS at the 5-minute evaluation point after the application of Surgicel ${ }^{\circledR}-\mathrm{P}$, without re-occurrence of bleeding needing other hemostatic treatment, until the initiation of fascia closure. Re-application of Surgicel ${ }^{\circledR}-P$ was allowed and did not affect subsequent effectiveness endpoints. The secondary effectiveness endpoints were the proportions of subjects achieving this criterium at 3 and 10 minutes following the initial Surgicel ${ }^{\circledR}-P$ application. The 5-minute primary endpoint has conventionally been employed in similar studies, $[14,18]$ and the secondary 3 - and 10-minute endpoints were included for comparison with broader preclinical and clinical experience with topical hemostats.[14, 17, 19-21] The investigators' evaluation of the ease-of-use of the Surgicel $\circledast$-P delivery system was investigated using a questionnaire survey.

\section{Safety Monitoring}

Investigators monitored AEs from the time of Surgicel®-P application through the 30-day and 6-month follow-up points and adjudicated all AEs for their relationship with study product and procedure. Specific safety endpoints were the incidence of thromboembolic events and TBS re-bleeding events requiring medical/surgical intervention through day 30 , and SAEs requiring surgical intervention through month 6 , which were considered to have an unlikely, possible, probable, or causal relationship to the study treatment. An internal independent Product Safety Committee reviewed cumulative safety data and adjudicated all events for the specific safety endpoints.

\section{Statistical Analysis}

Primary and secondary endpoints were analyzed using the intention-to-treat (ITT) set, which consisted of all subjects for whom a TBS was identified, and summarized descriptively [frequency count and percentage with 2-sided Clopper-Pearson 95\% confidence interval (CI)]; missing data were considered as failures. Analyses on the per-protocol (PP) set (evaluable set), which consisted of all ITT subjects who had no major protocol deviation affecting the primary endpoint and who had data available for this endpoint, were considered supportive. Effectiveness endpoints were also stratified by bleeding severity 
(mild or moderate) and surgical approach (open versus endoscopy). The primary endpoint was compared between surgical approaches using the Chi-Square test. Ease-of-Use questionnaire data were summarized descriptively. Safety endpoints were analyzed using the safety set, which consisted of all subjects who received study product, and summarized descriptively (frequency count and percentage); missing safety data were not imputed. Software SAS ${ }^{\circledR}$ version 9.4 or higher was used.

\section{Results}

\section{Subjects and Surgical Procedures}

Of the 132 subjects who provided informed consent, 29 subjects were screen failures for either intraoperative findings precluding study treatment (16 subjects), failing in- or exclusion criteria (12 subjects), and patient withdrawal prior to surgery (1 subject) (Figure 1). As a result, 103 subjects were included in the ITT set. A total of 100 subjects completed the study as planned, while 2 subjects died during followup prior to the 6-month visit and one subject was lost to follow-up. One subject, although treated, had a major protocol deviation due to failing to meet an inclusion criterion and was excluded from the PP set, which resulted in 102 evaluable subjects included in the PP set. There were 8 investigative sites, each having enrolled 2-20 evaluable subjects.

The median (range) age of the study population was 64.0 (33-88) years, the male/female gender distribution was 50.5\%/49.5\%, and subjects were predominantly Caucasian [93/103 (90.3\%)] (Table 1).

The distribution of operative procedures was urological (37.9\%), abdominal (32.0\%), gynecological (14.6\%), cardiothoracic (13.6\%) and pelvic (1.9\%); open surgery $(53.4 \%)$ was slightly more frequent than laparoscopic/thoracoscopic surgery (46.6\%) (including 7 robotic and 2 thoracoscopic procedures). The TBS tissue types included parenchymatous tissue (19.4\% with the majority (19/20) being liver), connective tissue (45.6\%) and a separate category of loose areolar tissue (27.2\%), as well as a small number of other TBS (7.8\%) (Table 1). The median surface area of the TBS was $4 \mathrm{~cm}^{2}$ (range $0.02-72.0$ ) and bleeding was considered mild in $72 / 103$ subjects (69.9\%) and moderate in the remaining $31 / 103$ subjects (30.1\%) (Table 1$)$.

In 100/103 subjects (97.1\%), one single Surgicel®-P applicator was used, and sufficient coverage was obtained in all subjects (100\%). At initial Surgicel®-P application, manual compression was applied in $28 / 102$ procedures $(27.5 \%, n=1$ data unavailable) (Figure 2). During longitudinal assessment of hemostasis, this rate declined progressively to $2 / 102$ procedures at fascia closure $(2 \%, n=1$ data unavailable), while the percentage of bleeding sites showing hemostasis increased from 89/103 procedures (86.4\%) at 3 minutes to $96 / 101$ procedures (95\%) at fascia closure (Figure 2 ). In 18 subjects $(17.5 \%)$, additional Surgicel®-P treatment was administered with/without manual compression or observation, whereas in 7 subjects (6.8\%), investigators reverted to standard-of-care using other hemostatic methods including cautery, suture, SURGICEL $®$-SNoW or TachoSil (Figure 2). 
The median total surgical procedure duration was $197 \mathrm{~min}$ (range 32-895 min), median total time in the operating room was $244 \mathrm{~min}$ (range 81-995 $\mathrm{min}$ ) and median total postoperative hospital stay was 5 nights (range 0-76 nights, data unavailable for $n=1$ ). The median estimated total intra-operative blood loss amounted to $150 \mathrm{~mL}$ (range $5-4,000 \mathrm{~mL}$ ), and blood and blood product transfusions were given in $13 / 103$ subjects $(12.6 \%)$.

Table 1. Subject Demographics, Surgical Procedure and Target Bleeding Site Characteristics. 


\section{Patient Characteristics}

Age at Consent, years

Mean (SD)

61.0

(12.8)

Median (range)

Gender, n (\%)

Male

Female

Race, n (\%)

Caucasian

Asian

2 (1.9)

Not reported

8 (7.8)

Ethnicity, n (\%)

Not Hispanic or Latino

91 (88.3)

Not reported

Prior and/or Concomitant Medical Conditions, n (\%)

Relevant Surgical History, n (\%)

\section{Surgical Procedure Characteristics}

Surgical Approach, n (\%)

Open

Laparoscopic/thoracoscopic

Surgical Procedure, n (\%)

Cardiothoracic $^{1}$

Abdominal

Pelvic 


\section{Target Bleeding Site Characteristics}

Primary Method for Hemostasis, n (\%)

None (other methods impractical)

Suture

Ligation

Cautery

Other

Type of Bleeding, n (\%)

Mild

$72(69.9)$

Moderate

$31(30.1)$

Tissue Type, $\mathrm{n}(\%)$

Connective tissue

$47(45.6)$

Loose Areolar tissue

$28(27.2)$

Parenchyma 20 (19.4)

Other $^{2}$

$8(7.8)$

Size (Area), $\mathrm{cm}^{2}$

Mean (SD)

Median (range)

${ }^{1} \mathrm{~N}=12(11.7 \%)$ cardiac and $\mathrm{n}=2$ lung $(1.9 \%)$

2 superior vena cava needle holes $(n=1)$, perivascular tissue $(n=1)$, right atrium needle holes $(n=1)$, epicardial dissection on left ventricle $(n=1)$, peri-adrenal tissue $(n=3)$, periprostatic tissue $(n=1)$

\section{Effectiveness Endpoints}

The primary endpoint analysis (Figure 3 ) in the ITT set showed the percentage of subjects achieving hemostatic success at 5 minutes to be $90 / 103$ subjects $(87.4 \% ; 95 \% \mathrm{Cl}[79.4 \%, 93.1 \%])$. The primary endpoint success rate according to bleeding intensity was $67 / 72$ subjects with mild bleeding $(93.1 \%$; $95 \% \mathrm{Cl}[84.5 \%, 97.7 \%])$ versus $23 / 31$ subjects with moderate bleeding $(74.2 \% ; 95 \% \mathrm{Cl}[55.4 \%, 88.1 \%])$. 
According to surgical approach, the success rate was $50 / 55$ subjects $(90.9 \% ; 95 \% \mathrm{Cl}[80.0 \%, 97.0 \%])$ for the open procedure group and $40 / 48$ subjects $(83.3 \%$; $95 \% \mathrm{Cl}[69.8 \%, 92.5 \%])$ for the laparoscopic/thoracoscopic procedure group ( $p=0.25$, Chi-square test). Endpoint analysis results in the PP set were similar (not shown).

The secondary endpoint analysis (Figure 3 ) in the overall group showed hemostatic success in 80/103 subjects $(77.7 \% ; 95 \% \mathrm{Cl}[68.4 \%-85.3 \%])$ at 3 minutes and in $95 / 103$ subjects $(92.2 \% ; 95 \% \mathrm{Cl}[85.3 \%$, $96.6 \%]$ ) at 10 minutes. In the subgroup analysis according to surgical approach, hemostatic success for the open procedure group was $44 / 55$ subjects $(80.0 \% ; 95 \% \mathrm{Cl}[67.0 \%, 89.6 \%])$ at 3 minutes and $53 / 55$ subjects $(96.4 \% ; 95 \% \mathrm{Cl}[87.5 \%, 99.6 \%])$ at 10 minutes; for the laparoscopic/thoracoscopic group, hemostatic success was $36 / 48$ subjects $(75.0 \% ; 95 \% \mathrm{Cl}[60.4 \%, 86.4 \%])$ at 3 minutes and $42 / 48$ subjects $(87.5 \%, 95 \% \mathrm{Cl}[74.8 \%, 95.3 \%])$ at 10 minutes. In the subgroup analysis according to bleeding severity, hemostatic success for mild bleeding was $61 / 72$ subjects $(84.7 \% ; 95 \% \mathrm{Cl}[74.3 \%, 92.1 \%]$ at 3 minutes and $69 / 72$ subjects $(95.8 \% ; 95 \% \mathrm{Cl}[88.3 \%, 99.1 \%])$ at 10 minutes; for moderate bleeding, hemostatic success was $19 / 31$ subjects $(61.3 \% ; 95 \% \mathrm{Cl}[42.2 \%, 78.2 \%])$ at 3 minutes and $26 / 31$ subjects $(83.9 \%, 95 \% \mathrm{Cl}$ $[66.3 \%, 94.6 \%])$ at 10 minutes.

Of note, for two subjects, the 10-minute hemostasis assessment was not performed as the fascia was closed at 5 minutes and 8 minutes; these subjects were considered as successes given the specific clinical bleeding scenarios with hemostatic success at 3 and 5 minutes without rebleeding or the subsequent need for additional treatment.

\section{Safety}

Over 6-month follow-up, a total of 257 AEs occurred in 70/103 subjects (68.0\%) (safety set), which included 33 SAE in 24 subjects (23.3\%) and 19 severe AEs in 15 subjects (14.6\%) (Table 2). There were no AEs or SAEs, specifically, no post-operative TBS-rebleeding or thrombo-embolic events, considered by the investigator to be unlikely, possibly, probably or causally related to Surgicelß-P. A total of 64 subjects (62.1\%) experienced at least one AE that was categorized as unlikely, possibly, probably or causally related to the study procedure. Seven subjects experienced AEs of ileus, which were considered anticipated for their procedure. All cases of ileus were dynamic/functional and resolved with non-surgical treatment and without sequalae. The AE and SAE occurred most frequently within the system classes of gastro-intestinal disorders (43/103 subjects [41.7\%]) and infections and infestations (27/103 subjects [26.2\%]). Two subjects (1.9\%), both known with pancreatic cancer, died between the day-30 and the month- 6 visit: one subject died at 3 months from an undetermined cause, and one subject died at 6 months due to pulmonary embolism after chemotherapy.

\section{Table 2. Adverse Events.}




\section{Surgicel®-P}

$\mathrm{N}=103$

\begin{tabular}{ll} 
Total Number of Adverse Events & 257 \\
\hline Total Number of Serious Adverse Events & 33 \\
\hline Number (\%) of Subjects with at Least 1 Event in the Category & \\
\hline AE & $70(68.0)$ \\
\hline Serious AE & $24(23.3)$ \\
\hline Severe AE & $15(14.6)$ \\
\hline AE Related* to Surgicel $8-P$ & $0(0.0)$ \\
\hline SAE Related* to Surgicel®-P & $0(0.0)$ \\
\hline AE Related* to Study Procedure & $64(62.1)$ \\
\hline Subjects with at Least One AE Expected/Anticipated & $55(53.4)$ \\
\hline Number (\%) of Subject Deaths & $2(1.9)$
\end{tabular}

*possibly related, probably related, or related

\section{Surgeon Ease-of-Use Questionnaire}

Data were collected for a total of 43 procedures, including 19 open and 22 laparoscopic and 2 thoracoscopic procedures (Supplementary Information 2). For the majority of procedures, investigators either 'agreed' or 'strongly agreed' that Surgicel $®-P$ was easy and quick to prepare for application $[41 / 43$ (95.3\%)] and could be easily applied to the TBS [39/43 (90.7\%)], that the investigator could exactly control the amount of Surgicel ${ }^{\circledR}-\mathrm{P}$ applied to the TBS $[30 / 43(69.8 \%)]$ and could adequately cover a broad area of bleeding [40/43 (93.0\%)], and that excess Surgicel®-P was easy to irrigate/aspirate out of surgical site without disturbing the clot at the TBS [29/36 (80.5\%)]. Similarly, for the majority of procedures, the investigators 'agreed' or 'strongly agreed' feeling confident about the ability of the product to achieve and maintain hemostasis [37/43 (86.1\%) and 37/43 86.1\%), respectively]. A subgroup analysis according to surgical approach (Figure 4) showed a similar response pattern for open and laparoscopic/thoracoscopic procedures; across the different survey items, the proportion of surgeons who 'strongly agreed' varied between $42.1 \%$ and $84.2 \%$ for the open approach (median $63.2 \%$ ), and between $28.6 \%$ and $54.2 \%$ for the laparoscopic/thoracoscopic approach (median $45.8 \%$ ) (Figure 4).

\section{Discussion}


This post-market clinical follow-up study shows that Surgicel ${ }^{\circledR}-\mathrm{P}$ is effective and safe in controlling mildto-moderate surgical bleeding in adults undergoing major general, gynecological, urological and cardiothoracic procedures. Hemostatic success and safety were demonstrated for both open and laparoscopic or thoracoscopic approaches, and surgeons favorably evaluated the use of Surgicel®-P and the delivery system. This real-world data supports the Surgicel®-P efficacy data from in vivo animal models and confirms its indication within the Surgicel ${ }^{\circledR}$ group of products for continuous bleeding on raw or broad surfaces, or anatomically complex surgical sites.[14, 17]

The observed 5-minute hemostatic success rate of $87.4 \%$ indicates that the adjuvant use of Surgicel®-P can control most appropriately selected mild-to-moderate bleeding sites and thus helps to avoid bystander damage from additional hemostatic measures such as ligature or cautery. Longitudinal evaluation showed that most of these bleeding sites were controlled earlier, at 3 minutes. Manual compression, which was initially applied in approximately one third of bleeding sites, was almost completely abandoned within 10 minutes, when hemostatic success exceeded $90 \%$. These data confirm that Surgicel $\circledast$-P exhibits equal or superior hemostatic performance to other Surgicel ${ }^{\circledR}$ formulations used for similar indications. $[19,20,22]$ Expectedly, the 5-minute hemostatic success rate was lower for moderate $(74.2 \%)$ than mild (93.1\%) bleeding sites. Although the optimal choice of a topical hemostat is primarily dependent on the surgical scenario and the agent's mechanism of action, bleeding intensity critically determines time to hemostasis. However, secondary endpoint analyses at earlier and later time points showed that over time, the difference between mild and moderate bleeding sites declined, with both reaching more than $80 \%$ hemostatic control within 10 minutes. This is consistent with findings in the animal models where Surgicel ${ }^{\circledR}-\mathrm{P}$ was shown to be effective for both mild and moderate bleeding from liver punch biopsies or liver abrasions.[17] Within the 10-minute time frame of the study, approximately one fifth of procedures required additional treatment, but this consisted mostly of repeat application of Surgicel ${ }^{\circledR}$-P. Subsequent reversion to standard of care was deemed necessary in only $6.8 \%$ of procedures, further confirming the efficacy of Surgicel ${ }^{\circledR}-\mathrm{P}$ for the selected indications. Success rates for laparosopic/thoracoscopic procedures and open procedures showed no statistically significant difference, and the ease-of-use survey demonstrated similar agreement profiles for both surgical approaches.

Procedure time and procedural blood loss were captured for the entire surgical procedure. Although median values were consistent with the nature of the procedures, these parameters, along with hospital length-of-stay, showed a high upper end-of-range value due to 1 subject who experienced surgical complications unrelated to the TBS. In this patient, who underwent a laparotomy for a retroperitoneal tumor resection, the target bleeding site exhibited moderate bleeding on the mesentery and showed 5minute hemostatic success with no re-bleeding requiring additional treatment. Also, the maximal procedure time of 895 minutes and hospital length-of-stay of 76 days were observed in a single patient who underwent a Whipple pancreaticoduodenectomy and whose target bleeding site was a mildly bleeding area of the liver parenchyma that showed effective hemostasis for all primary and secondary endpoints. 
For the large majority of the procedures, surgeons indicated in the ease-of-use survey that they either agreed or strongly agreed that Surgicel ${ }^{\circledR}-\mathrm{P}$ was easy and quick to prepare and could adequately cover a broad area of bleeding, and that they felt confident in the ability of the product to achieve and maintain hemostasis at the TBS. Similar to its fabric forms, the powdered Surgicel $®-P$ is resorbable, but it is recommended to apply the least amount necessary to obtain hemostasis, and to remove any excess powder when bleeding is controlled. In $97.1 \%$ of the procedures, a single applicator was used while most surgeons agreed that this allowed adequate coverage of the bleeding site and that excess product could be easily removed. Considering that two thirds of bleeding sites were mild and one third was moderate, this real-world data confirm that the Surgicel $\AA_{-P} P$ applicator is adequately dosed for its clinical indication.

Whereas appropriately dosed Surgicel $\circledast$-P product is essentially resorbed within 2-5 weeks, follow-up in this post-market study was extended to 6 months to monitor longer-term complications. Although infrequent, complications of ORC use have been reported. Most concern inflammatory foreign body reactions with granuloma or pseudotumor formation, which are thought to be related to the characteristic acidic environment created by ORC, particularly if excess product is present.[10, 23] In addition, mass effect complications have been described due to ORC swelling, resulting, for example, in neural compression syndromes.[10, 24] As per manufacturer's instructions, in this study, investigators were advised to irrigate or aspirate excess powder once hemostasis was achieved, while not disturbing the clot. During the extended 6-month follow-up, no safety signals were identified, supporting the safety of the adjunctive use of Surgicel $\circledast$-P. The AEs and SAEs reported were those anticipated following the distinct major surgical procedures in the study, in an adult population with high rates of pre-existing medical and surgical histories. While several AEs were considered to have a possible causal relationship with the procedure, such was not the case for a relationship with the Surgicel®-P device. Specifically, there were no SAEs, post-operative re-bleeding or thromboembolic events assessed as having a causal relationship (including unlikely or at least possible) to Surgicel ${ }^{\circledR}-\mathrm{P}$.

Because of its single-arm design, the study lacks comparative evaluation of efficacy and safety with other hemostatic agents. However, the longitudinal assessment of hemostasis and the prospective 6month clinical follow-up in an adequately sized patient sample and broad range of major surgical procedures with open and laparoscopic/thoracoscopic approaches provide a robust set of real-world data that validate the efficacy and safety of the product.

Oxidized regenerated cellulose hemostats are particularly useful for surgical sites that show continuous bleeding from broad or raw surfaces, and that is unlikely to stop with conventional methods. Retrospective studies have shown that appropriate use of ORC is associated with reduced hemostatic agent use and hemostat spending relative to the use of other hemostats, while leaving clinical outcome unaffected.[25] Within the Surgicel $\circledast$ family of products, the use of the advanced fibrillar and SNoW forms were associated with a shorter length of stay and lower all-cause cost than the original sheerwoven Surgicel $\circledast$ product.[26] While these advanced Surgicel ${ }^{\circledR}-\mathrm{P}$ products share a superior hemostasis performance, the powder structure of Surgicel ${ }^{\circledR}-\mathrm{P}$ also offers improved conformability and adherence to broad surfaces and anatomically complex wound geometry. A recent comparative study in porcine liver 
and spleen bleeding models showed both powder and sponge gelatin-based mechanical hemostats to be effective for parenchymal surgical bleeding, but powder to be more suitable for mild diffuse bleeding.[27]

\section{Conclusion}

The findings of this post-market clinical follow-up study support the safety and efficacy of the new powder form of oxidized regenerated cellulose, Surgicel ${ }^{\circledR}-\mathrm{P}$ in controlling mild to moderate parenchymal surgical bleeding in a broad range of surgical procedures. The design of Surgicel $₫$-P applicators is intuitive and easy to use offering a controlled and consistent expression of powder regardless of device orientation.

\section{Abbreviations}

$\mathrm{AE}$, Adverse Event

$\mathrm{Cl}$, Confidence Interval

ICH, International Conference on Harmonization

IFU, Instructions for Use

INR, International Normalized Ratio

ITT, Intent to Treat

MedDRA, Medical Dictionary for Regulatory Activities

NA, n/a, Not Applicable

ORC, Oxidized regenerated cellulose

PP, Per Protocol

SAE, Serious Adverse Event

THA, topical hemostatic agent

\section{Declarations}

Ethics approval and consent to participate. The study was performed in accordance with the ICH tripartite guideline for Good Clinical Practice (1996) and the Declaration of Helsinki (2013). Protocols and informed consent forms were approved by the institutional review boards at participating sites (WoSRES (West of Scotland Research Ethics Service) (Paisley, United Kingdom), Commissie Voor Medische Ethiek 
(Gent, Belgium), Ziekenhuis Oost Limburg Ethisch comité (Genk, Belgium). Patients were considered for enrollment only if informed consent was obtained.

Consent for publication. Not applicable

Availability of data and materials. All data generated or analyzed during this study are included in this published article and its supplementary information files, and at https://clinicaltrials.gov/ct2/show/results/NCT03762200.

Competing interests. The study was funded by Ethicon, Inc. Golden Jubilee National Hospital (NA), Ziekenhuis Oost-Limburg (ED) and University Hospital of Ghent (FB) were in receipt of financial support from Ethicon, Inc. for conduct of this research project. RK, IB, EB, are employees of Ethicon Inc.

Funding. Financial support for the study was provided by Ethicon, Inc. Ethicon, Inc. contributed to the design of the study, analysis, and interpretation of data and reviewed the manuscript.

Authors' contributions.

NA, ED, RK, IB, EB, FB designed the study. NA, FB, ED collected data. NA, ED, RK, IB, EB, FB analyzed and interpreted data. NA, ED, RK, IB, EB, FB provided input, reviewed and approved the final manuscript for submission to BMC Surgery.

Acknowledgements. We gratefully acknowledge the investigators and subinvestigators for their care for the patients, and Piet Hinoul for contributing to the trial design. Medical writing support, in the form of literature, medical writing and editorial services was provided by An Billiau, MD PhD, Celsus Medical Writing, and funded by Ethicon Inc.

\section{References}

1. Tompeck AJ, Gajdhar AUR, Dowling M, Johnson SB, Barie PS, Winchell RJ et al. A comprehensive review of topical hemostatic agents: The good, the bad, and the novel. J Trauma Acute Care Surg. 2020;88(1):e1-e21.

2. Wright JD, Ananth CV, Lewin SN, Burke WM, Siddiq Z, Neugut Al et al. Patterns of use of hemostatic agents in patients undergoing major surgery. J Surg Res. 2014;186(1):458-66.

3. Johnston SS, Jamous N, Mistry S, Jain S, Gangoli G, Danker W et al. Association of In-Hospital Surgical Bleeding Events with Prolonged Hospital Length of Stay, Days Spent in Critical Care, Complications, and Mortality: A Retrospective Cohort Study Among Patients Undergoing NeoplasmDirected Surgeries in English Hospitals. Clinicoecon Outcomes Res. 2021;13:19-29.

4. Corral M, Ferko N, Hollmann S, Broder MS, Chang E. Health and economic outcomes associated with uncontrolled surgical bleeding: a retrospective analysis of the Premier Perspectives Database. Clinicoecon Outcomes Res. 2015;7:409-21. 
5. Stokes ME, Ye X, Shah M, Mercaldi K, Reynolds MW, Rupnow MF et al. Impact of bleeding-related complications and/or blood product transfusions on hospital costs in inpatient surgical patients. BMC Health Serv Res. 2011;11:135.

6. Ye X, Lafuma A, Torreton E, Arnaud A. Incidence and costs of bleeding-related complications in French hospitals following surgery for various diagnoses. BMC Health Serv Res. 2013;13:186.

7. Al-Attar N, Johnston S, Jamous N, Mistry S, Ghosh E, Gangoli G et al. Impact of bleeding complications on length of stay and critical care utilization in cardiac surgery patients in England. $\mathrm{J}$ Cardiothorac Surg. 2019;14(1):64.

8. Corral M, Ferko N, Hogan A, Hollmann SS, Gangoli G, Jamous N et al. A hospital cost analysis of a fibrin sealant patch in soft tissue and hepatic surgical bleeding. Clinicoecon Outcomes Res. 2016;8:507-19.

9. Ferko N, Danker W. Hemostat Utilization training program achieves cost savings and utilization efficiency. Healthcare Purchasing News. 2017:34-35.

10. Chiara O, Cimbanassi S, Bellanova G, Chiarugi M, Mingoli A, Olivero G et al. A systematic review on the use of topical hemostats in trauma and emergency surgery. BMC Surg. 2018;18(1):68.

11. Lewis KM, Spazierer D, Urban MD, Lin L, Redl H, Goppelt A. Comparison of regenerated and nonregenerated oxidized cellulose hemostatic agents. Eur Surg. 2013;45:213-20.

12. Hutchinson RW, george K, Johns D, Craven L, Zhang G, Shnoda P. Hemostatic efficacy and tissue reaction of oxidized regenerated cellulose hemostats. Cellulose. 2013;20:9.

13. Spangler D, Rothenburger S, Nguyen K, Jampani H, Weiss S, Bhende S. In vitro antimicrobial activity of oxidized regenerated cellulose against antibiotic-resistant microorganisms. Surg Infect (Larchmt). 2003;4(3):255-62.

14. MacDonald MH, Tasse L, Wang D, Zhang G, De Leon H, Kocharian R. Evaluation of the Hemostatic Efficacy of Two Powdered Topical Absorbable Hemostats Using a Porcine Liver Abrasion Model of Mild to Moderate Bleeding. J Invest Surg. 2020:1-9.

15. Surgicel ${ }^{\circledR}$ Powder (oxidized regenerated cellulose) - Instructions for Use [https://www.jnjmedicaldevices.com/en-US/product/ethicon-surgicel-powder]

16. Wang AY, Rafalko J, MacDonald M, Ming X, Kocharian R. Absorbable Hemostatic Aggregates. ACS Biomater Sci Eng. 2017;3(12):3675-86.

17. MacDonald MH, Wang AY, Clymer JW, Hutchinson RW, Kocharian R. An in vivo comparison of the efficacy of hemostatic powders, using two porcine bleeding models. Med Devices (Auckl). 2017;10:273-79.

18. ARISTA ${ }^{\mathrm{TM}}$ AH Absorable Hemostatic Particles - Instructions for Use [https://www.bd.com/assets/documents/pdh/initial/arista-ah-ifu.pdf]

19. Fischer CP, Wood CG, Shen J, Batiller J, Hart JC, Patel B et al. A randomized trial of aprotinin-free fibrin sealant versus absorbable hemostat. Clin Appl Thromb Hemost. 2011;17(6):572-7. 
20. Genyk Y, Kato T, Pomposelli JJ, Wright JK, Jr., Sher LS, Tetens V et al. Fibrin Sealant Patch (TachoSil) vs Oxidized Regenerated Cellulose Patch (Surgicel Original) for the Secondary Treatment of Local Bleeding in Patients Undergoing Hepatic Resection: A Randomized Controlled Trial. J Am Coll Surg. 2016;222(3):261-8.

21. Matonick JP, Hammond J. Hemostatic efficacy of EVARREST, Fibrin Sealant Patch vs. TachoSil(R) in a heparinized swine spleen incision model. J Invest Surg. 2014;27(6):360-5.

22. Fischer CP, Bochicchio G, Shen J, Patel B, Batiller J, Hart JC. A prospective, randomized, controlled trial of the efficacy and safety of fibrin pad as an adjunct to control soft tissue bleeding during abdominal, retroperitoneal, pelvic, and thoracic surgery. J Am Coll Surg. 2013;217(3):385-93.

23. Agarwal MM, Mandal AK, Agarwal S, Lal A, Prakash M, Mavuduru R et al. Surgicel granuloma: unusual cause of "recurrent" mass lesion after laparoscopic nephron-sparing surgery for renal cell carcinoma. Urology. 2010;76(2):334-5.

24. Menovsky T, Plazier M, Rasschaert R, Maas Al, Parizel PM, Verbeke S. Massive swelling of Surgicel(R) Fibrillar hemostat after spinal surgery. Case report and a review of the literature. Minim Invasive Neurosurg. 2011;54(5-6):257-9.

25. Martyn D, Meckley LM, Miyasato G, Lim S, Riebman JB, Kocharian R et al. Variation in hospital resource use and cost among surgical procedures using topical absorbable hemostats. Clinicoecon Outcomes Res. 2015;7:567-74.

26. Martyn D, Kocharian R, Lim S, Meckley LM, Miyasato G, Prifti K et al. Reduction in hospital costs and resource consumption associated with the use of advanced topical hemostats during inpatient procedures. J Med Econ. 2015;18(6):474-81.

27. Paterno VA, Bisin A, Addis A. Comparison of the efficacy of five standard topical hemostats: a study in porcine liver and spleen models of surgical bleeding. BMC Surg. 2020;20(1):215.

\section{Figures}




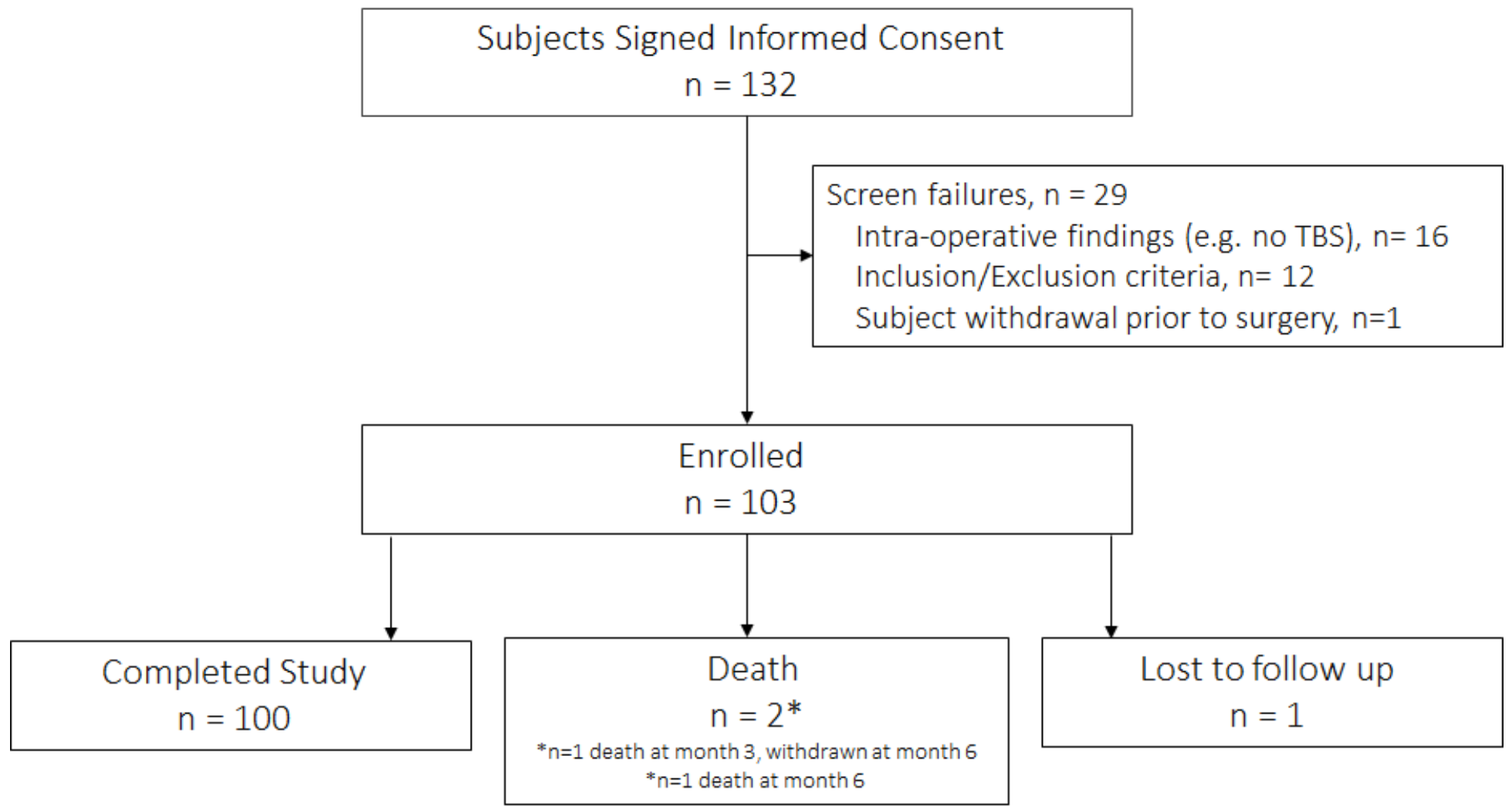

Figure 1

Disposition of Study Subjects.

a.

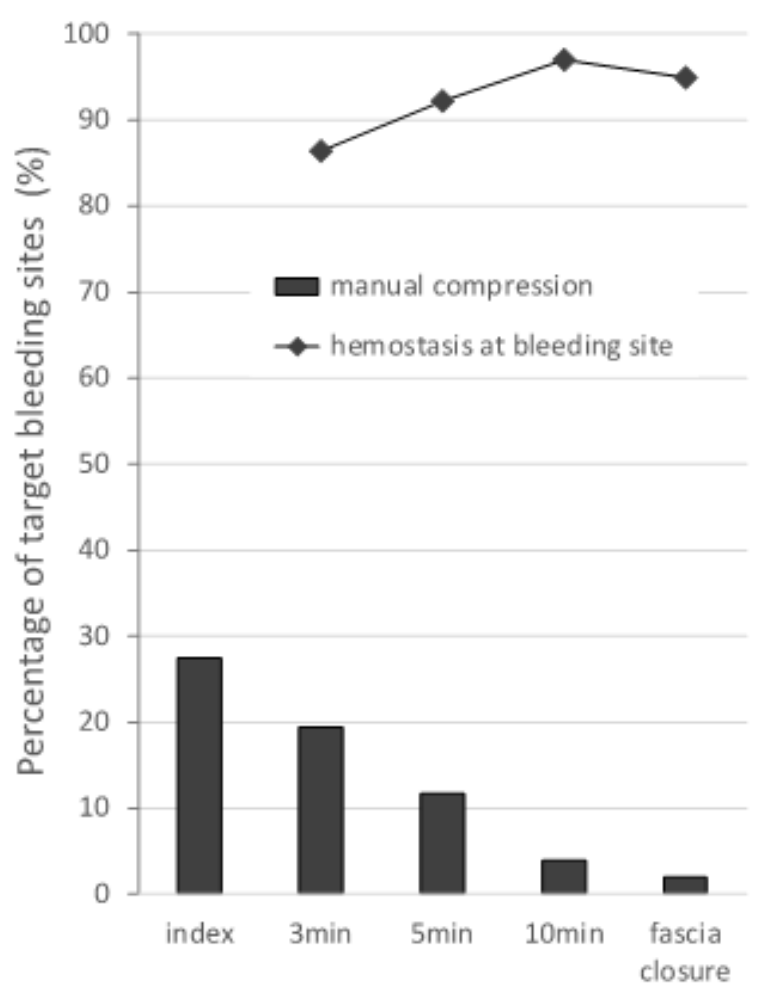

Time after initial SURGICEL ${ }^{\oplus}$-P b.

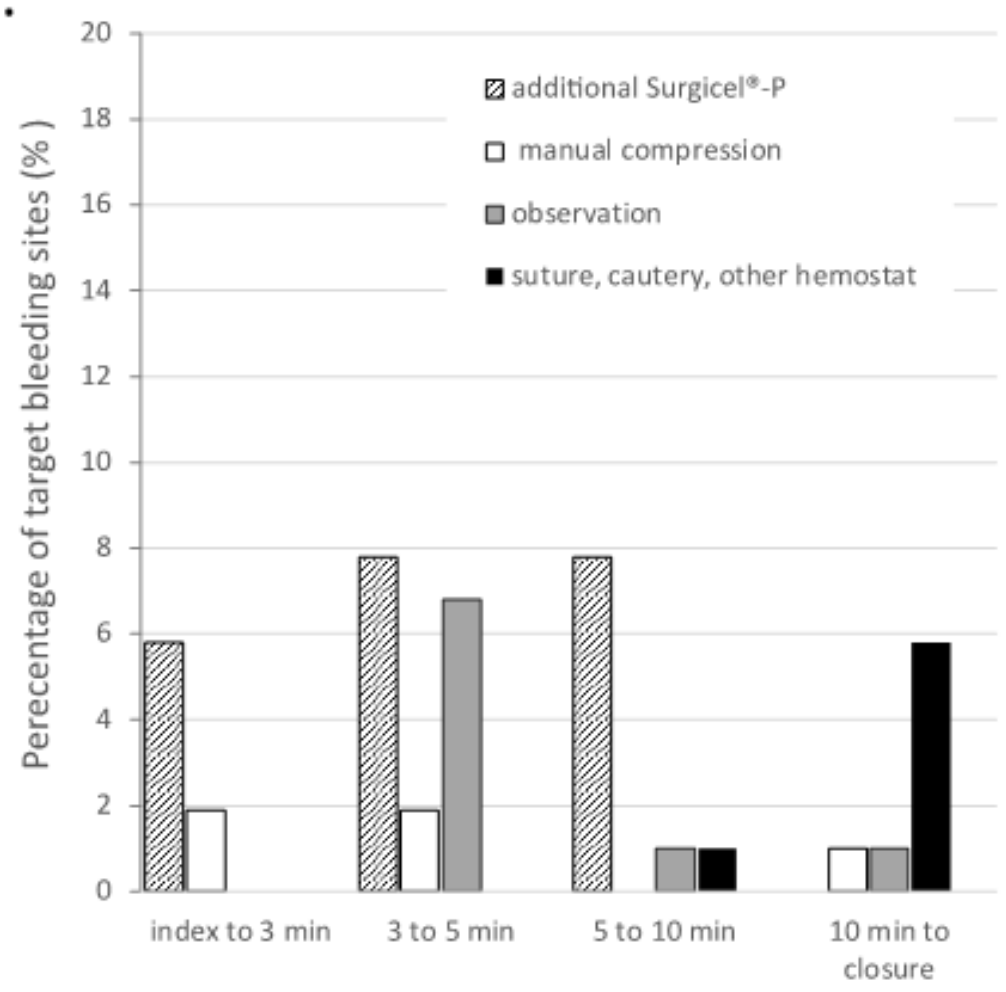

Time after initial SURGICEL ${ }^{\circledast}-\mathrm{P}$ 
Figure 2

Evaluation of Hemostasis and Need for Additional Hemostatic Treatment. Shown are (a) the percentage of procedures where manual compression was applied to the target bleeding site at index (along with Surgicel $\circledast$-P application) and during evaluation of hemostasis until fascia closure, along with the percentage of target bleeding sites that were considered hemostatic at 3,5 and 10 minutes and before fascia closure; (b) the percentage of procedures where additional hemostatic measures were applied to the target bleeding site after the first Surgicel ${ }^{\circledR}-\mathrm{P}$ application at index. Values represent percentages of target bleeding sites.

a.

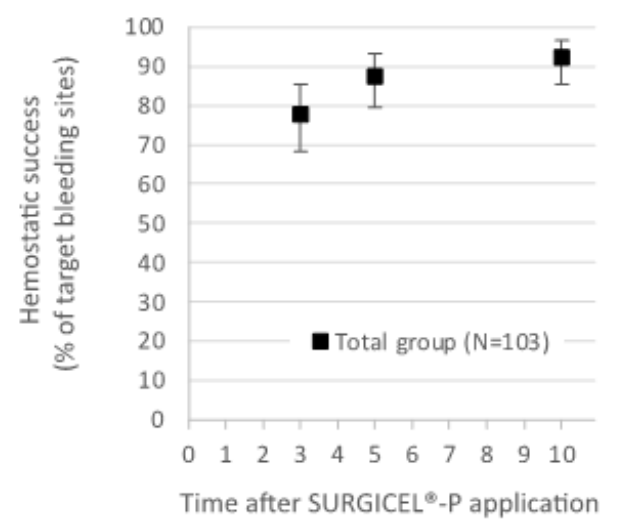

b.

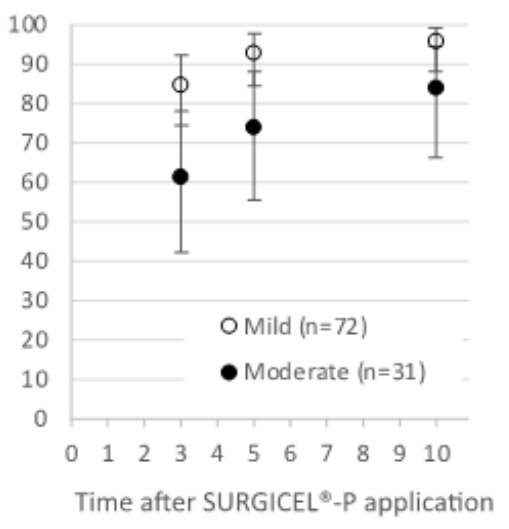

C.

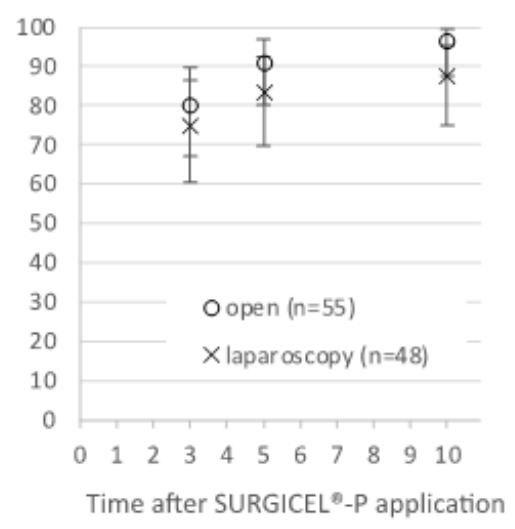

\section{Figure 3}

Primary and Secondary Hemostatic Efficacy Endpoints (ITT Set). Shown are the primary and secondary study endpoints for hemostatic efficacy for (a) the total group, and for the subgroups according to (b) bleeding intensity (mild or moderate) and (c) surgical approach (open versus laparoscopic/thoracoscopic surgery). Values indicate percentages (\%) of target bleeding sites, with $95 \%$ confidence intervals. 


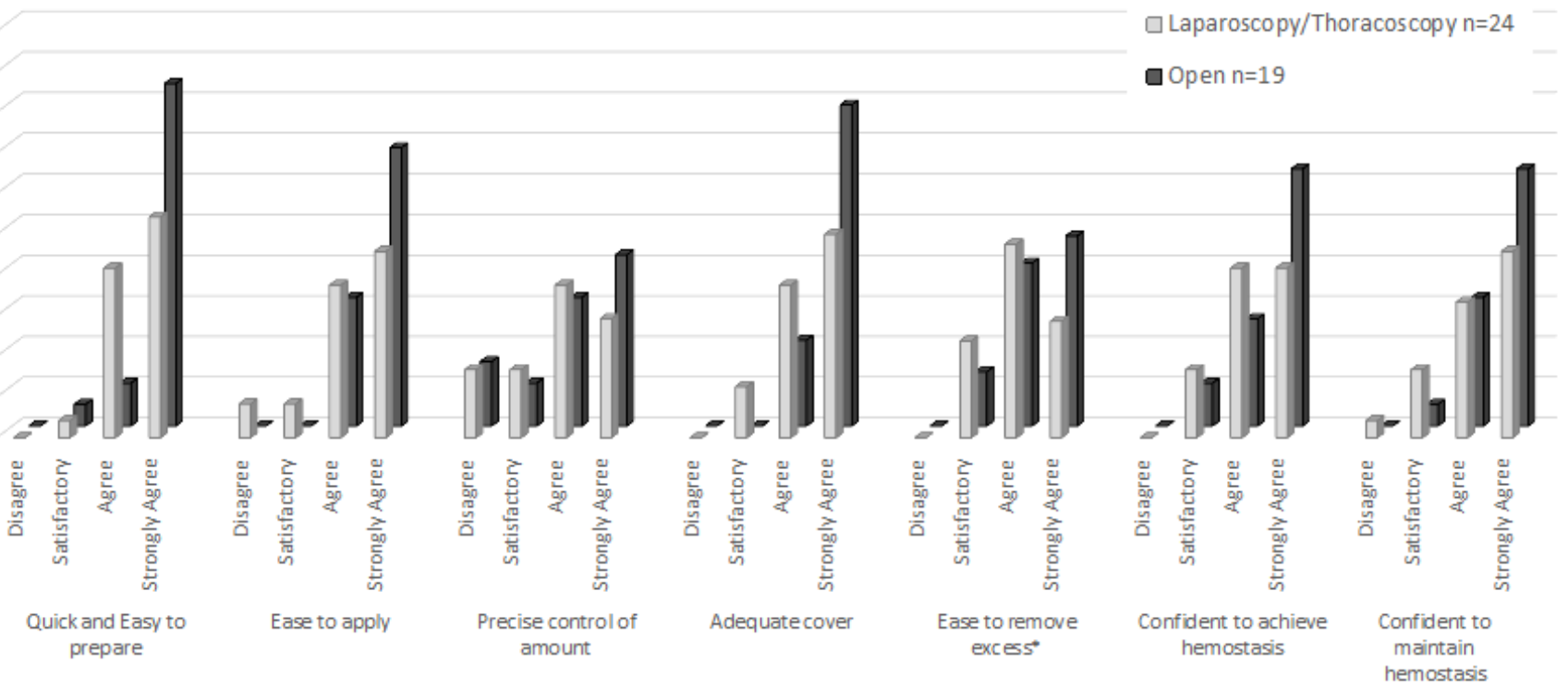

Survey questions

Figure 4

Ease of Use Survey. Shown are the responses to the 7-item ease-of-use questionnaire, as reported by each surgeon for their first 2 open and first 2 laparoscopic/thoracoscopic procedures performed within this study. Values represent the percentage of procedures for which the indicated agreement score was reported. Results are shown according to surgical approach (open versus laparoscopic/thoracoscopic procedure). ${ }^{*} \mathrm{n}=15$ for open procedures and $\mathrm{n}=21$ for laparoscopy/thoracoscopy procedure.

\section{Supplementary Files}

This is a list of supplementary files associated with this preprint. Click to download.

- AlAttaretal.SurgicelPManuscriptBMCSurgerySupplementaryinformation.docx 